\title{
Design and Simulation of IoT Systems Using the Cisco Packet Tracer
}

\author{
Norman Gwangwava ${ }^{1}$, Tinashe B. Mubvirwi ${ }^{2}$ \\ ${ }^{1}$ Mechanical, Energy, and Industrial Engineering, Botswana International University of Science and Technology, Palapye, \\ Botswana \\ ${ }^{2}$ Sable Chemical Industries Limited, Kwekwe, Zimbabwe \\ Email: ^gwangwavan@biust.ac.bw
}

How to cite this paper: Gwangwava, N and Mubvirwi, T.B. (2021) Design and Simulation of IoT Systems Using the Cisco Packet Tracer. Advances in Internet of Things, 11, 59-76.

https://doi.org/10.4236/ait.2021.112005

Received: February 19, 2021

Accepted: April 16, 2021

Published: April 19, 2021

Copyright () 2021 by author(s) and Scientific Research Publishing Inc. This work is licensed under the Creative Commons Attribution International License (CC BY 4.0).

http://creativecommons.org/licenses/by/4.0/

(c) (i) Open Access

\begin{abstract}
Design and implementation of Internet of Things (IoT) systems require platforms with smart things and components. Two dominant architectural approaches for developing IoT systems are mashup-based and model-based approaches. Mashup approaches use existing services and are mainly suitable for less critical, personalized applications. Web development tools are widely used in mashup approaches. Model-based techniques describe a system on a higher level of abstraction, resulting in very expressive modelling of systems. The article uses Cisco packet tracer 7.2 version, which consists of four subcategories of smart things-home, smart city, industrial and power grid, to design an IoT based control system for a fertilizer manufacturing plant. The packet tracer also consists of boards-microcontrollers (MCU-PT), and single boarded computers (SBC-PT), as well as actuators and sensors. The model facilitates flexible communication opportunities among things-machines, databases, and Human Machine Interfaces (HMIs). Implementation of the IoT system brings finer process control as the operating conditions are monitored online and are broadcasted to all stakeholders in real-time for quicker action on deviations. The model developed focuses on three process plants; steam raising, nitric acid, and ammonium nitrate plants. Key process parameters are saturated steam temperature, fuel flowrates, $\mathrm{CO}$ and $\mathrm{SO}_{\mathrm{x}}$ emissions, converter head temperature, $\mathrm{NO}_{\mathrm{x}}$ emissions, neutralisation temperature, solution temperature, and evaporator steam pressure. The parameters need to be monitored in order to ensure quality, safety, and efficiency. Through the Cisco packet tracer platform, a use case, physical layout, network layout, IoT layout, configuration, and simulation interface were developed.
\end{abstract}

\section{Keywords}

Internet of Things (IoT), Smart Sensors, Wireless Sensors, Process Control, 
Cisco Packet Tracer, Simulation, Smart Factory, Cloud Computing

\section{Introduction}

Internet of things (IoT) and Internet of Services (IoS) concepts are a major part of the broad industry 4.0 technologies. These interconnected things and services enable modern smart factories and integrated value chains to function optimally. Today's connected and data driven world is disruptive and gives businesses a competitive advantage. Ubiquitous cloud services enable the deployment of IoT applications anywhere and offer complete control. IoT technologies will ensure systems established in Industry 4.0 are of low cost and have lean operating systems. Industry players must be prepared for unprecedented changes that IoT brings [1] [2] [3] [4]. IoT technologies such as RFID, wired and wireless sensor networks, and embedded systems enable the digitization and virtualization of shared resources and capabilities in the services and manufacturing industries for access through the cloud. Mell and Grance [5] define Cloud Computing (CC) as a model for enabling ubiquitous, convenient, on-demand network access to a shared pool of configurable computing resources (e.g., networks, servers, storage, applications, and services) that can be rapidly provisioned and released with minimal management effort or service provider interaction. The cloud is mainly categorized as private cloud, community cloud, public cloud and hybrid cloud [6]. Today's business space is characterized by smart environments/spaces and self-aware things such as smart transport, products, cities, buildings, rural areas, energy, health systems, wholesale and retail outlets.

The spread of real-time data across companies-given the availability of appropriate analytical tools and methods - can have a significant impact on the entire company. Organizations that use IoT, digitization, and big data technologies have been evaluated as having a higher level of logistic service, more efficient processes with their partners, improved cooperation between certain logistic functions, and higher market and financial performance and competitiveness [7]. Countries that promote the use of high technology achieve more efficient production processes, which lead to better productivity and economies of scale. The German Ministry of Education and Research established Industry 4.0 as a roadmap to promote the German high-tech industry and its strategy [8] [9]. The ubiquitous computing era presents enterprises with huge quantities of data, known as Big Data. The data is gathered through smart sensors among physically networked objects.

Although there is much hype about IoT and related technologies, practical rollout still remains depressed. There are many underlying reasons for the mismatch between what exists on the ground and the promises being made. Adoption of new unknown technologies is a risky activity and is currently expensive [10]. Lack of creation and consistent implementation of a corporate digital 
strategy hampers the rollout of industry 4.0 solutions [11]. The growth of IoT poses a great security threat. Cybersecurity concerns are for the device, data, and the individuals [12] [13]. The engineering of IoT systems is another challenge for industry practitioners responsible for design and deployment. Challenging aspects which they face include safe programming and validation, achievement of resilience and graceful degrading, as well as the development of new tools and methods [14]. IoT infrastructure and ecosystem should promote reusability, interoperability integration, modular programming, better flexibility, agility and, ease of maintenance. Internet connectivity is crucial for the success of IoT technologies in industry. "Any Thing" and "Every Thing" should be interconnected with the global information and communication infrastructure. This can be achieved through network accessibility (getting on a network) and compatibility (common ability to consume and produce data) [15]. Although the internet protocol version 6 (IPv6) was introduced to solve the problem of the shortage of IP addresses experienced with IPv4, its global implementation has challenges. Each connected device and "being" requires a unique IP address, which makes the network complex and difficult to manage, all the connected sensors need to be powered, and parallel management of different protocols and legacy assets during the transition period is a complex task [16].

The main objective of the article is to advance research in the development and implementation of IoT systems. The research is a case study of a fertilizer manufacturing plant. Needs requirements for an IoT platform were established and a Visual Programming Language (VPL) platform for design and simulating the solution was identified. Several solutions that use VPLs for developing IoT-based systems exist, but a section criterion was used to select the most suitable platform. Cisco Packet Tracer 7.2 was chosen for developing the IoT platform because it offers a variety of network components that simulate a real network. The tool offers a variety of IoT functionalities, accommodates smart devices, components, sensors, actuators, and can simulate micro-controllers such as Arudino or Raspberry Pi [17]. The article bridges the gap on IoT development and deployment. It lays out a quick rollout strategy by using a digital platform that has inbuilt IoT objects and programming capabilities.

The structure of the article has a literature review in Section 2. The literature covers the definition, architecture, capabilities, and enabling technologies for IoT. It also looks at visual programming for IoT systems and the extended perspective of IoT, which is the Internet of Everything (IoE). Section 3 covers the methodology used to design and simulate the IoT platform for the fertilizer manufacturing company, the results and discussion. Section 4 concludes the article.

\section{Literature Review}

This section reviews literature on standard definitions for IoT, associated technologies, layered architecture, extended view of IoT, i.e. IoE, and lastly visual programming languages (VPLs). 


\subsection{Internet of Things (IoT) Definition and Technologies}

The definition of IoT continues to evolve. IoT represents electrical or electronic devices, of varying sizes and capabilities, which are connected to the Internet. The scope of the connections has grown beyond just machine-to-machine communication (M2M) to broadly focused machine-to-people (M2P), and people-to-people (P2P) communications [18] [19]. M2M is mainly being utilized to implement "smart factories" using IP networks to inter-connect physical infrastructure with sensors, which results in extra capabilities such as analytics and monitoring using technologies such as radio frequency identification (RFID). M2P is used to capture and analyze consumer data to be used in designing products and services such as mobile marketing to push the manufacture-consumer relationship as close to the consumer as possible. P2P utilizes converged network services such as real-time video collaboration tools with "Bring Your Own Device" (BYOD) capabilities [20]. IoT can also be defined as a system of interrelated computing devices, mechanical and digital machines, objects, animals, or people that are provided with unique identifiers and the ability to transfer data over a network without requiring human-to-human or human-to-computer interaction [21] [22]. I-Scoop [23] define the Internet of Things as a network of connected devices with: 1) unique identifiers in the form of an IP address which 2) have embedded technologies or are equipped with technologies that enable them to sense, gather data and communicate about the environment in which they reside and/or themselves. Cisco coins its definition of IoT as, "the intelligent connectivity of smart devices, expected to drive massive gains in efficiency, business growth and quality of life" [24] [25].

Things in "IoT" can refer to anything which possesses smart characteristics, such as sensors, embedded chip, automobile, people, animal, agricultural produce or anything in the value chain, road infrastructure, building or anything in the built environment, consumer goods, plant equipment or machinery, and many others. When these "Things" are provided with unique identifiers (UIDs), they gain the ability to transfer data over a network with no need for human-to-human or human-to-computer interaction. "Things" equipped with UIDs can sense each other and communicate, can be accessed and interacted with over the Internet. Specific functionality that is aided by these features is remote access for monitoring, configuration, and troubleshooting, and data analysis. This changes how, where, and who makes decisions in the modern data-driven world. IoT helps private and public enterprises to find more operating efficiencies, deliver greater value to customers, employees, and citizens in general, and enable new business models. Each IoT device provides capabilities-features or functions-it can use on its own or in conjunction with other IoT and non-IoT devices to achieve one or more goals [12]. These capabilities are provided in Table 1.

IoT devices employ a broad array of networking protocols, applications and network domains [19] [26] [27] [28]. 6LoWPAN (IPv6 over Low-Power Wireless 
Table 1. IoT device capabilities.

\begin{tabular}{|c|c|c|}
\hline Capability Type & \multicolumn{2}{|l|}{ Description } \\
\hline \multirow[t]{3}{*}{$\begin{array}{l}\text { Transducer } \\
\text { Capabilities: }\end{array}$} & \multicolumn{2}{|c|}{$\begin{array}{l}\text { Provide the ability for computing devices to interact directly with physical entities } \\
\text { of interest. Every IoT device has at least one transducer capability. The two types } \\
\text { of transducer capabilities are given below: }\end{array}$} \\
\hline & Sensing: & $\begin{array}{l}\text { The ability to provide an observation of an aspect of the } \\
\text { physical world in the form of measurement data. E.g. } \\
\text { temperature measurement, radiographic imaging, optical } \\
\text { sensing, and audio sensing. }\end{array}$ \\
\hline & Actuating: & $\begin{array}{l}\text { The ability to change something in the physical world. } \\
\text { E.g. heating coils, cardiac electric shock delivery, } \\
\text { electronic door locks, servo motors, and robotic arms. }\end{array}$ \\
\hline \multirow[t]{4}{*}{$\begin{array}{l}\text { Interface } \\
\text { capabilities: }\end{array}$} & \multicolumn{2}{|c|}{$\begin{array}{l}\text { Enable device interactions (e.g., device-to-device communications, } \\
\text { human-to-device communications). Examples are: }\end{array}$} \\
\hline & Application interface: & $\begin{array}{l}\text { The ability for other computing devices to communicate } \\
\text { with an IoT device through an IoT device application. } \\
\text { E.g. an application programming interface (API). }\end{array}$ \\
\hline & Human user interface: & $\begin{array}{l}\text { The ability for an IoT device and people to communicate } \\
\text { directly with each other. E.g. touch screens, haptic } \\
\text { devices, microphones, cameras, and speakers. }\end{array}$ \\
\hline & Network interface: & $\begin{array}{l}\text { The ability to interface with a communication network } \\
\text { for the purpose of communicating data, i.e. to use a } \\
\text { communication network. Includes both hardware and } \\
\text { software (e.g., a network interface card or chip and the } \\
\text { software implementation of the networking). E.g. } \\
\text { Ethernet, Wi-Fi, Bluetooth, Long-Term Evolution (LTE), } \\
\text { and ZigBee. }\end{array}$ \\
\hline \multirow[t]{4}{*}{$\begin{array}{l}\text { Supporting } \\
\text { capabilities: }\end{array}$} & \multicolumn{2}{|c|}{ Provide functionality that supports the other IoT capabilities. Examples are: } \\
\hline & \multicolumn{2}{|l|}{ Device management } \\
\hline & \multicolumn{2}{|l|}{ Cybersecurity } \\
\hline & \multicolumn{2}{|l|}{ Privacy capabilities } \\
\hline
\end{tabular}

Personal Area Networks) is an open standard defined by the Internet Engineering Task Force (IETF). The 6LoWPAN standard enables any low-power radio to communicate to the internet, including 804.15.4, Bluetooth Low Energy and Z-Wave (for home automation) [29] [30]. LiteOS is a Unix-like operating system for wireless sensor networks. LiteOS supports smartphones, wearables, intelligent manufacturing applications, smart homes and Internet of Vehicles (IoV). The operating system also serves as a smart device development platform. AMQP (Advanced Message Queuing Protocol) is an open-source published standard for asynchronous messaging by wire. AMQP enables encrypted and interoperable messaging between organizations and applications. The protocol is used in client/server messaging and in IoT device management [31]. The broad range of enabling technologies for the IoT can be grouped into three categories: 1) technologies that enable "Things" to acquire contextual information, 2) technologies that enable "Things" to process contextual information, and 3) tech- 
nologies to improve security and privacy [32]. Laeeq and Shamsi [33] present a detailed classification of IoT technologies as shown in Table 2.

\subsection{Internet of Things (IoT) Architecture}

IoT architecture serves to illustrate how various technologies relate to each other and to communicate the scalability, modularity and configuration of IoT deployments in different scenarios [15]. Several architectures have been proposed since the inception of the IoT concept, which evolved from the 3-layered to the 5-layered architecture, service-oriented IoT-A architecture to the special purpose BeTaaS, Open IoT and IoT@Work architectures [33] [34]. The 3-layer architecture model is considered as a primary model for IoT work, which consists of the application layer, network layer, and perception layer. The task of Perception layer is to identify objects along with the collection of information. The 5-layer architecture is a more well-defined architecture and highly recommended for the IoT use. It consists of 2 additional layers as compared to 3-layer architecture that are Processing and Business layers. The processing layer is used to store all the data received from the transport layer (also called network layer) and process it. The processing layer uses technologies like cloud computing, and ubiquitous computing. Management of tasks (applications management and business modeling) is performed in the business layer [35] [36] [37]. Figure 1 shows the detailed five (5) layer IoT architecture. The architecture is pyramid shaped to resemble the plant automation pyramid.

\subsection{Internet of Everything (IoE)}

The term "Internet of Everything" was used by Cisco since the year 2012, but later on dropped at the dominance of IoT as the preferred term [38]. The Internet of Everything (IoE) brings together people, process, data, and things to make

Table 2. IoT technologies [32].

\begin{tabular}{|c|c|}
\hline IoT Category & Technologies \\
\hline \multicolumn{2}{|l|}{ Communication } \\
\hline Short Range: & NFC, RFID, ANT, Bluetooth, ZigBee, ZWave, IEEE802.15.4, WiFi \\
\hline Medium Range: & WiMAX, Weightless, DASH7, EnOcean, PLC, QR Code, Ethernet \\
\hline Large Range: & GPRS, GSM, GPS, 3G/ 4G, LTE, Satellite \\
\hline \multicolumn{2}{|c|}{ Prototype Hardware } \\
\hline & $\begin{array}{l}\text { Raspberry Pi, Hackberry, Arduino Yun, Arduino Uno, PCDuino, The Rascal, } \\
\text { Cubie Board, BeagleBone Black, OpenPicus Flyport WiFi, Pinoccio }\end{array}$ \\
\hline \multicolumn{2}{|l|}{ Operating System } \\
\hline & $\begin{array}{c}\text { Tiny OS, Contiki, Mantis, Nano-RK, LiteOS, FreeRTOS, Riot OS, } \\
\text { SNAP OS, Abacus OS, Sapphire OS }\end{array}$ \\
\hline \multicolumn{2}{|l|}{ Protocol: } \\
\hline & $\begin{array}{c}\text { REST, IPV6, 6LOWPAN, UDP, Chirp, DTLS, XMPP-IoT, } \\
\text { SSI, NanoIP, MQTT }\end{array}$ \\
\hline
\end{tabular}




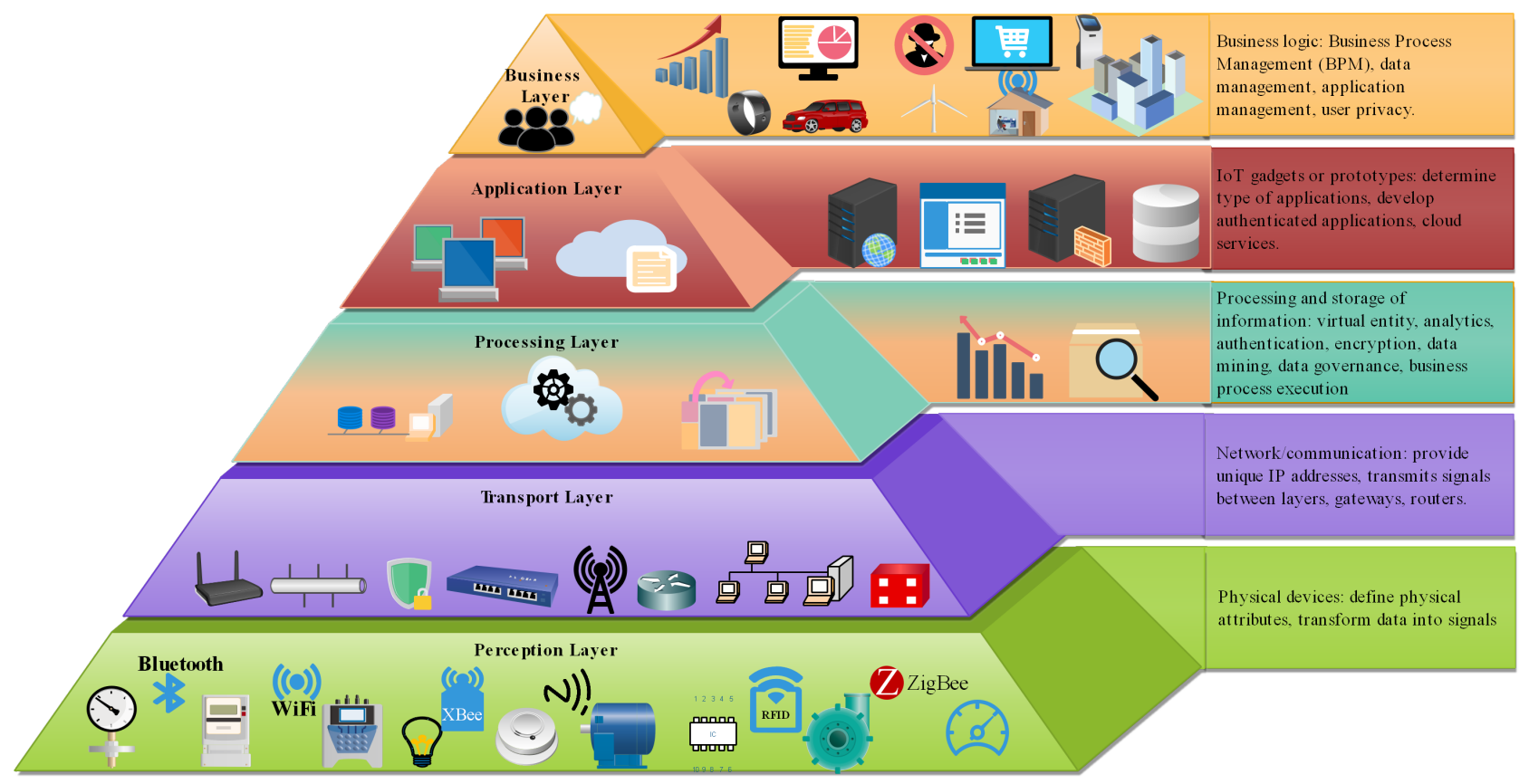

Figure 1. Five (5) layer IoT architecture.

networked connections more relevant and valuable than ever before-turning information into actions that create new capabilities, richer experiences, and unprecedented economic opportunity for businesses, individuals, and countries [39] [40]. There are many other distinguishing terms used in the field of IoT to refer to specific use cases. Some popular terms in use today are the Industrial IoT (IIoT), Consumer IoT (CIoT), Web of Things (WoT), and many others. Figure 2 depicts the combined IoE, IIoT, and CIoT.

Many modern consumer electronic devices, which are also present in organizations' facilities, are now connected IoT devices-kitchen appliances (refrigerators, microwave ovens, cooking stoves, etc), thermostats, home security cameras, door locks, light bulbs, and TVs. CIoT is used to refer to applications and uses cases to track personal "assets" - (asset tracking), such as pets, and skateboards, connected "smart home appliances" such as connected refrigerators, washing machines, light bulbs, etc [23]. Industrial Internet of Things (IIoT) describes typical industry use cases across a range of sectors such as manufacturing industries or utilities, smart cities and smart metering [41]. Web of Things (WoT) has been used to describe approaches to facilitate services offered at Open Systems Interconnection model (OSI)'s application layer [42].

\subsection{Visual Programming and Deployment of IoT}

In order to realize the full potential of IoT, there is a need to integrate ubiquitous smart devices and cloud based applications [43]. A combined IoT framework with a cloud at the center, gives the flexibility of dividing associated costs in the most logical manner and is also highly scalable. In the combined framework, sensing service providers can join the network and offer their data using a 


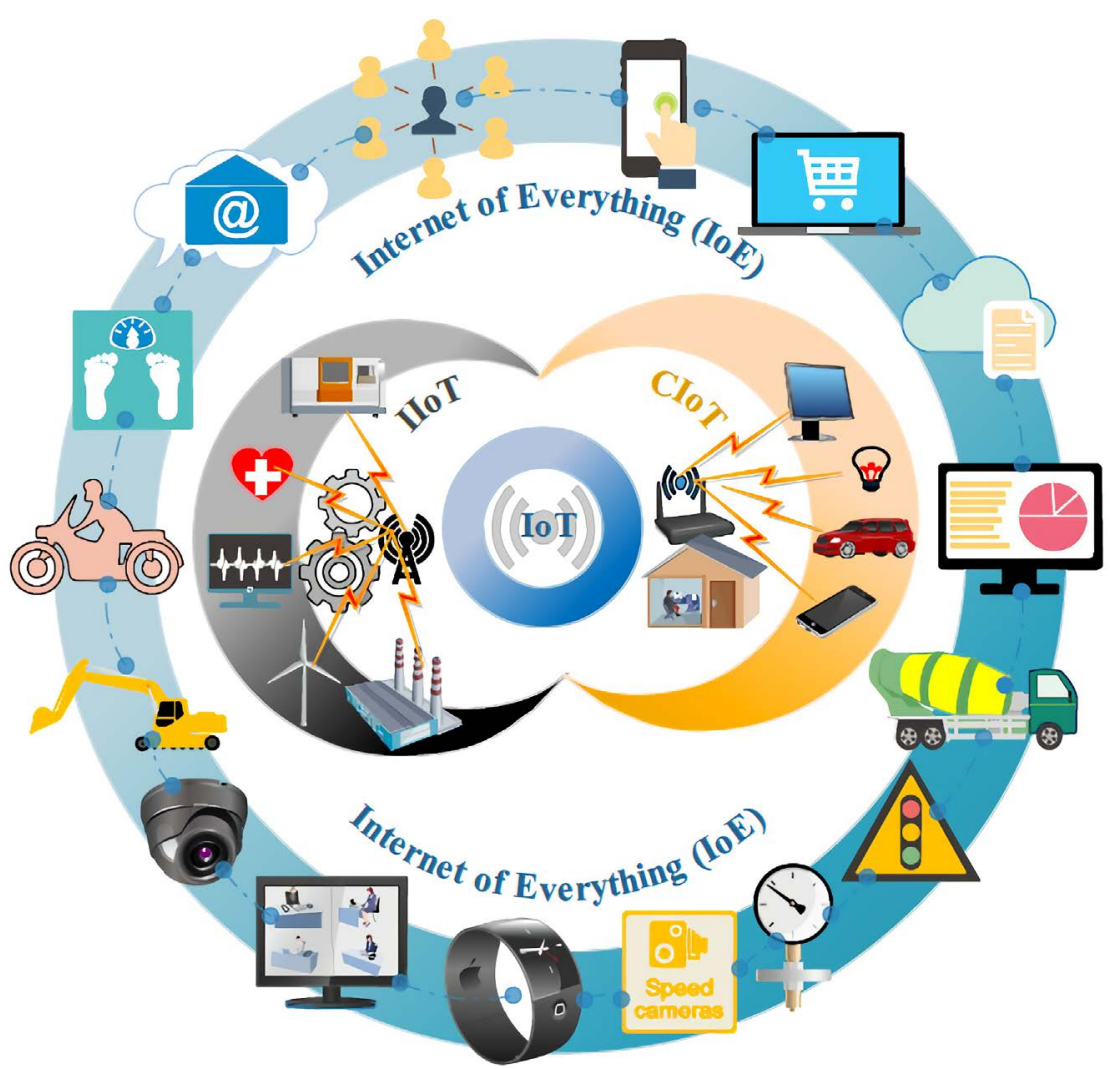

Figure 2. Internet of everything (IoE), industrial IoT (IIoT) and consumer IoT (CIoT).

storage cloud; analytic tool developers can provide their software tools; artificial intelligence experts can provide their data mining and machine learning tools useful in converting information to knowledge and computer graphics designers can offer a variety of visualization tools [22]. Cloud computing (CC) can offer these services through various models - Infrastructure as a Service (IaaS), Platform as a Service (PaaS), or Software as a Service (SaaS). This allows the full potential of human creativity to be tapped. Major players in the CC marketplace and related products are IBM, HP, Intel (Intel IoT Solution Alliance), Microsoft (Azure, NET, Node.js, Java, PHP), Google (Google App Engine-Python, Java, Go), Amazon (Elastic Cloud Compute-EC2, AWS, Simple Storage ServiceS3), Cisco, Kaa IoT, ThingWorx, ThingSpeak, and many others [44]. Availability of IoT application specific frameworks for rapid creation of applications and their deployment on cloud infrastructures is the key for achieving rapid development. Using such a framework, the developer of IoT applications will be able to harness the power of $\mathrm{CC}$ without knowing low-level details of creating reliable and scale applications [45].

A variety of low cost programmable hardware platforms have gained popularity, enabling communities and individuals to fast track their learning in IoT development and deployment. Most popular and cheapest platforms are Raspberry PI, Arduino Uno, Beagleboard, Intel Galileo, ESP8266, Particle, NXP, Android Things, and others [19]. These platforms are modular and flexible tools for not 
only real world applications but also educational purposes. A do-it-yourself (DIY) intuitive programming interface is required to enable the masses to interact with and customize the behavior of remote IoT devices on the Internet [46].

Several visual programming languages (VPLs) have been developed to help people start programming without immense knowledge about programming languages (i.e., expression, statement, loop clause, and functional orientation) [47] [48]. A VPL is any programming language that lets the user create programs by manipulating program elements graphically (allowing programming with visual expressions, a graphical user interface, spatial arrangement of graphic symbols, a drag-and-drop approach, moving some code blocks that execute a simple piece of logic, etc.) rather than by specifying them textually. VPLs are key tools for further enhancement, progress, and motivation towards developers in this field of IoT while reducing time-to-market in product (IoT-software/hardware) development life cycle [49] [50]. VPL solutions are mostly distinct, having a different focus, set of features and base themselves in different paradigms. Dias and Ferreira [39] give a comprehensive overview comparison and discussion of the VPL tools available for IoT. Examples of VPLs for IoT are Cisco Packet Tracer, Node-RED, Flogo, NETLab Toolkit (NTK), ArduBlock, Visuino, and many other tools. Designers of IoT applications should be relieved of most of heterogeneity and specifics (wide range of hardware and software entities running on specific platforms, middleware specific features, computing resources and protocols) [51]. Device heterogeneity is an intrinsic characteristic of any IoT system [52] [53]. An IoT platform must support different levels of device heterogeneity and abstract the device complexity to some extent. They need to use an integrated development environment (IDE) based on domain specific high level language which in its entities would abstract most of these intricacies and specifics. VPLs should have capability to support large scale design of IoT systems by combining several design blocks and saving them in application libraries, importing, reconfiguring and setting parameters with ease for new tags and locations, enabling reusability and scalability [54] [55].

\section{Methodology}

The article seeks to explore short development life cycle for IoT projects using a set of cloud based platforms and VPLs. A practical use case for a fertilizer manufacturing company was chosen. The company was commissioned in 1969 and since then, the technology for production of ammonium fertilizer evolved to include new technologies. The focus of the study is to automate the factory process systems and implement an IoT system to help manage the process for better process control and monitoring of key parameters through the use of smart wireless sensors which are able to communicate to the internet. Cisco Packet Tracer 7.2 was used for developing the IoT platform because it offers a variety of network components that simulate a real network. Cisco packet tracer enables developers to view the flow of data packets and carry out analysis on the data 
packets transmitted in the IoT network. All the IoT devices on Cisco Packet Tracer can be run on standard programs or can be customized by programming them with Java, Phyton or Blockly.

\subsection{Company Process Flow}

The company's overall process flow diagram is shown in Figure 3. Key process plants that need to be interlinked together are the steam raising plant (boiler house for producing dry steam at 13 bars using coal fired boilers), nitric acid plant (produces 57\% nitric acid for use in the production of ammonium nitrate), and ammonium nitrate plant (exothermic neutralization of nitric acid and ammonia). The smart factory use case is illustrated in Figure 4. Major process parameters that need to be monitored for the three plants are saturated steam temperature, converter head temperature, and neutralisation temperature respectively.

\subsection{IoT System Development}

When creating IoT simulations, it is advisable to utilize different physical layers in order to be able to adjust the environment variable to influence the IoT devices' behaviour. Figure 5 shows the physical separation of the smart fertilizer manufacturing factory, where three containers are used to physically split the network.

The network for the IoT system is logically separated into three areas: factory (shopfloor sensors), ISP servers (main control centre) and streets (end user devices), as shown in Figure 6. All IoT devices for the smart factory are connected to the router in the control centre. Factory floor sensors are wired to the microcontroller, which is in turn wirelessly connected to the home gateway. The control

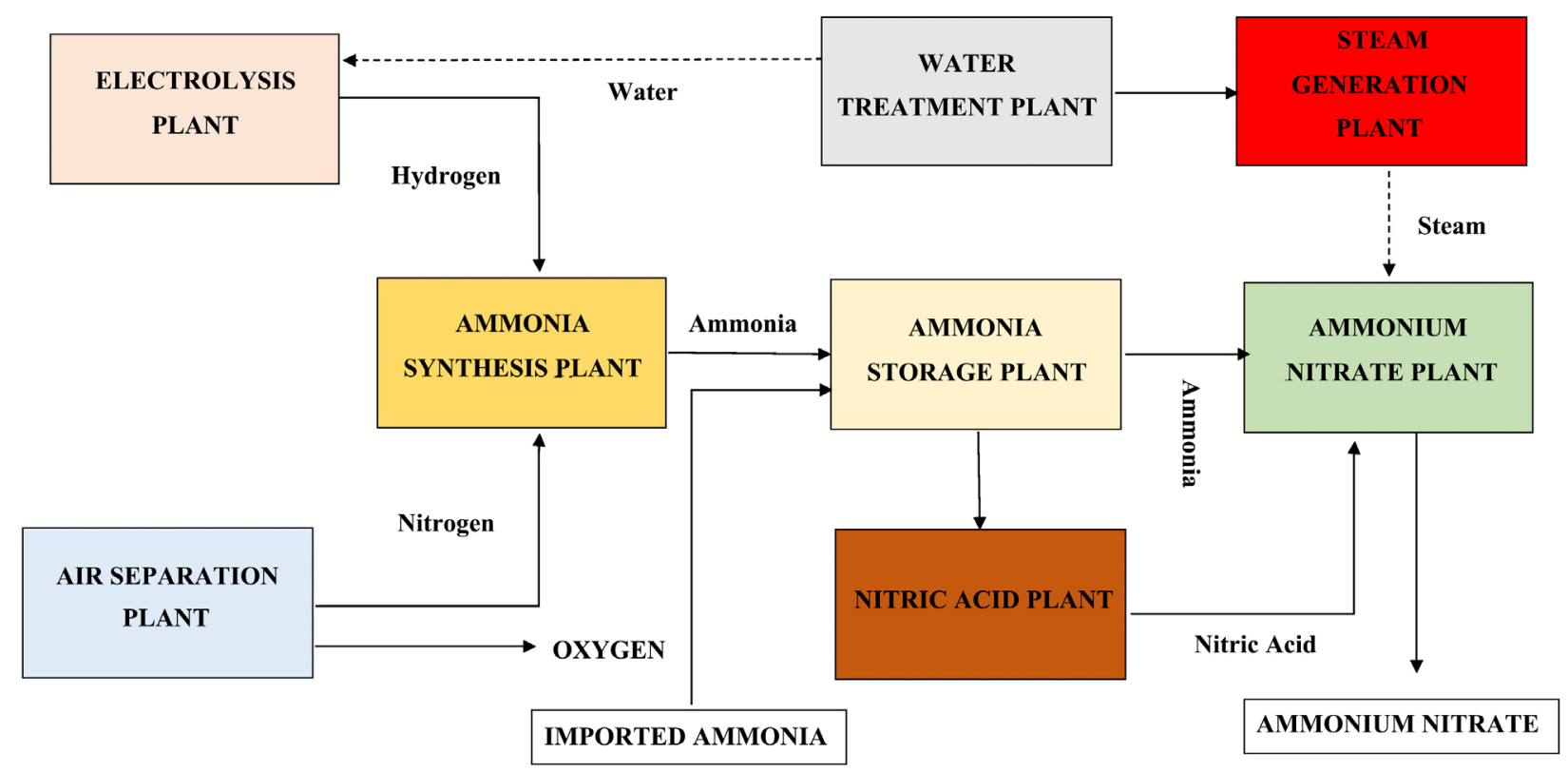

Figure 3. Process flow diagram the fertilizer plant. 


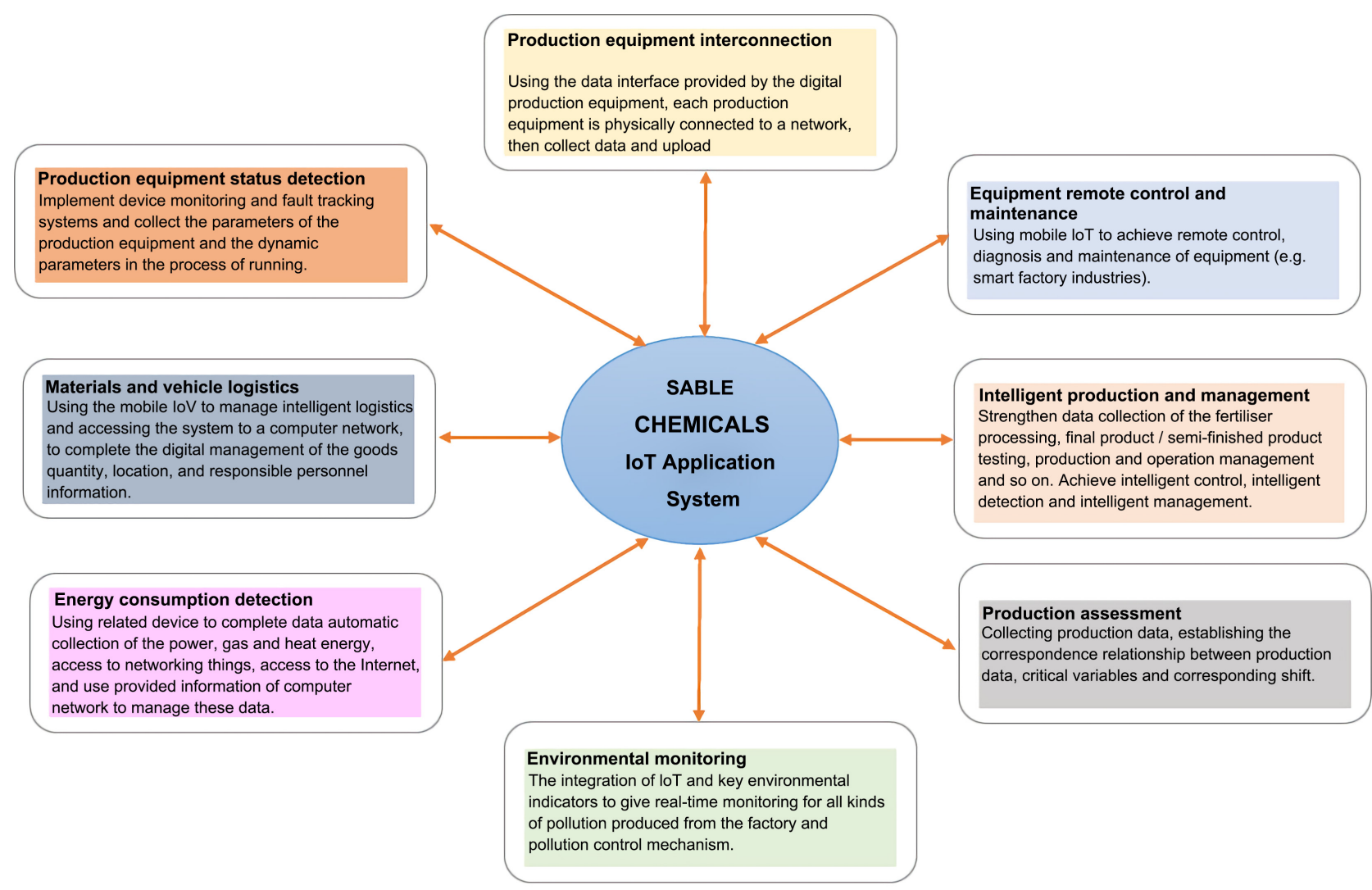

Figure 4. IoT application use cases for the fertilizer manufacturing plant.

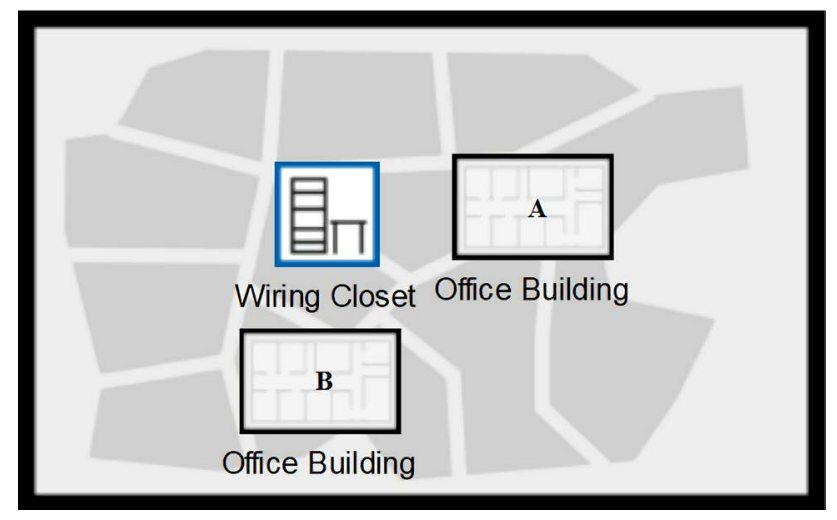

Figure 5. Physical layout.

centre is equipped with display interfaces for visual display of sensor readings. The home gateway broadcasts the data to the cloud via a DSL modem. The data is archived in servers and can be accessed by end user devices through the cell tower and wireless connections.

Internet settings in the router were the wireless Service Set IDentifier (SSID) and password. The same SSID, password and Dynamic Host Configuration Protocol (DHCP) default settings were used across all wireless devices, whilst the local server used static IP. Static IP addresses ensure that, in the event that the WLAN router is rebooted, the server IP remains the same, and there won't be 


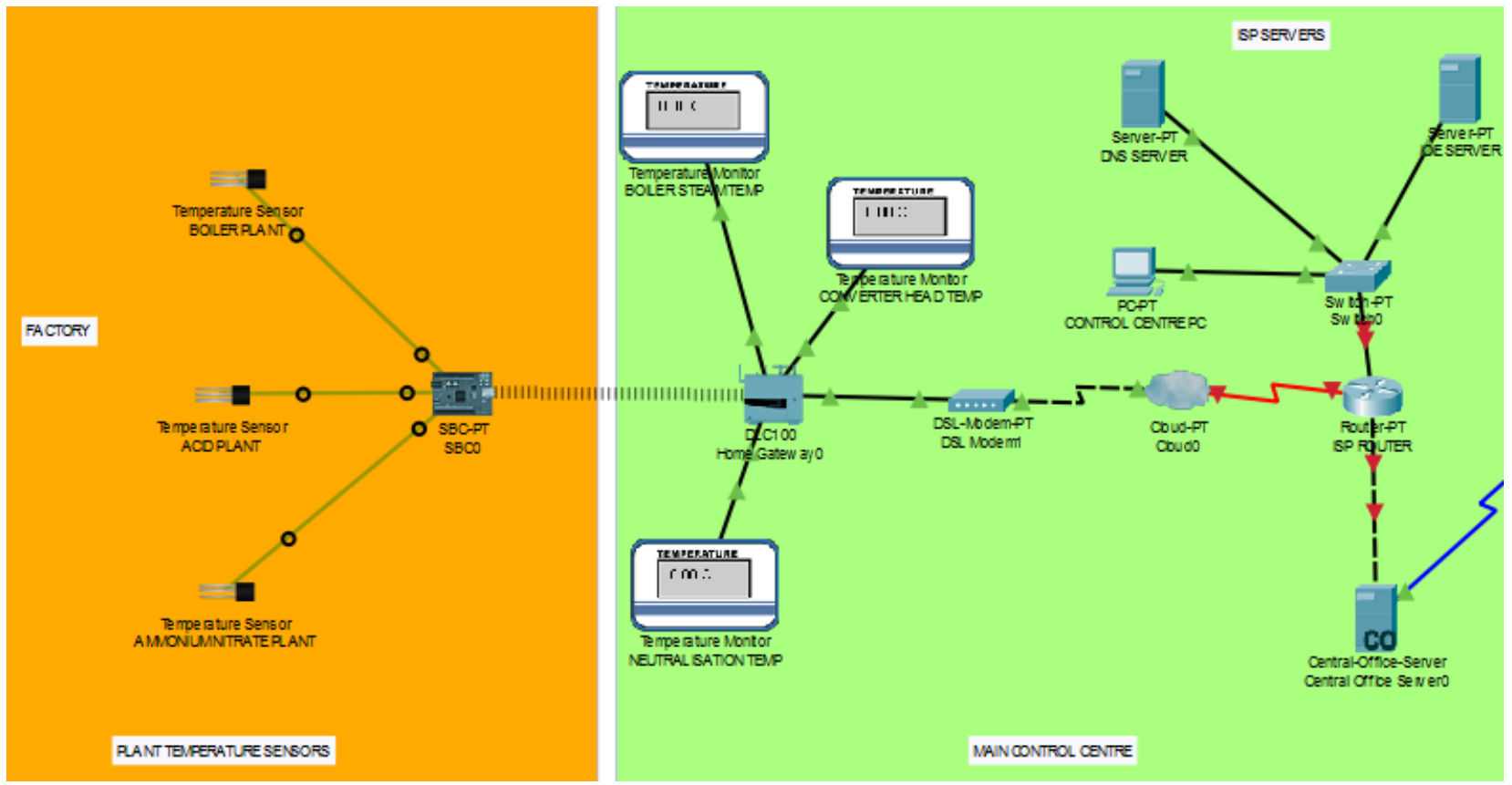

Figure 6. Network Layout.

any need to re-configure the devices assigning new IoT server IPs. The central office server provides DHCP services, IoT and DNS functionalities. These functionalities ensure that there is backend intelligence to the IoT simulation as well as facilitating hosting of the IoT homepage where end users could connect and interact with the smart factory. The DNS service helps in translating the IoT homepage URL into the IoT server IP.

The smart devices are remotely connected to the IoT backend server. The IoT connection enables the users to check the status of the IoT devices from an IoT browser homepage. The IoT browser homepage shows a list of the smart devices, allows visualization of their status, and also permits remote interaction with the devices. Logical interaction between smart devices can also be set while connected to the IoT main homepage. Interactions between devices are based on set conditions, such as starting the chiller when temperature of a particular unit needs to be lowered, or reducing oxygen supply to the boiler in order to reduce the fire tube temperatures. The central control PC, manager smartphones and tablets, also connected to the local central office wireless LAN (WLAN), can connect to the dedicated IoT homepage via a browser in order to monitor all connected IoT devices.

Cisco Packet Tracer has a feature with the possibility to switch from a real time to a simulation mode. The first mode enabled the possibility to create the underneath network, connect IoT devices and define IoT backend logic. However, only in the simulation mode, it was possible to validate that the network communication layer really happened between the devices. In the simulation mode it was in fact possible to simulate packet traffic between nodes and devices in order to check the connectivity, routing protocols and other network logic. 
This mode helped to physically visualize and troubleshoot any kind of network, for example setting up pings, or more complex packages between nodes. Figure 7 shows the simulation panel, whilst Figure 8 shows the real time flow of data packets.

\begin{tabular}{|c|c|c|c|c|c|}
\hline \multicolumn{5}{|c|}{ Simulation Panel } & $x$ \\
\hline \multicolumn{6}{|l|}{ Event List } \\
\hline \multirow[t]{13}{*}{ Vis. } & Time(sec) & Last Device & At Device & Type & \multirow[t]{12}{*}{4} \\
\hline & 9.772 & Home Gateway0 & BOILER STE... & STP & \\
\hline & 10.193 & - & ACID PLANT & IoT & \\
\hline & 10.194 & ACID PLANT & SBCO & loT & \\
\hline & 10.208 & - & AMMONIUM ... & І0T & \\
\hline & 10.209 & AMMONIUM NI... & $\mathrm{SBCO}$ & I0T & \\
\hline & 10.220 & - & BOILER PLANT & І०T & \\
\hline & 10.221 & BOILER PLANT & $\mathrm{SBCO}$ & І०T & \\
\hline & 11.295 & - & ACID PLANT & І0T & \\
\hline & 11.296 & ACID PLANT & $\mathrm{SBCO}$ & loT & \\
\hline & 11.313 & - & AMMONIUM ... & ІІT & \\
\hline & 11.314 & AMMONIUM NI... & $S B C O$ & 10T & \\
\hline & 11.326 & - & BOILER PLANT & loT & \multirow{2}{*}{$\equiv$} \\
\hline 9 & 11.327 & BOILER PLANT & $\mathrm{SBCO}$ & $10 \mathrm{~T}$ & \\
\hline \multicolumn{3}{|c|}{ Reset Simulation $\square$ Constant Delay } & & & $\begin{array}{r}\text { Captured to: } \\
11.327 \mathrm{~s} \\
\end{array}$ \\
\hline \multicolumn{2}{|c|}{ Play Controls } & & & & \\
\hline
\end{tabular}

Figure 7. Simulation panel.

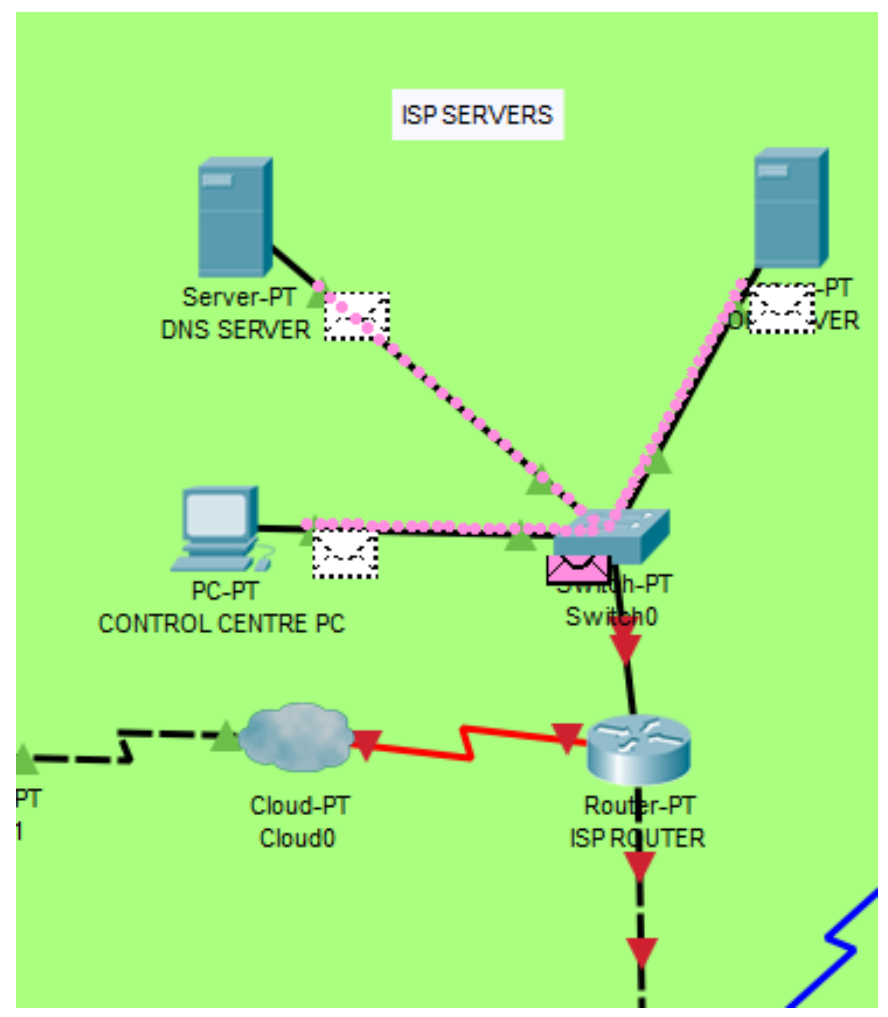

Figure 8. Real-time flow of data packets. 


\section{Conclusion}

The article reviewed the literature on IoT under the headings: standard definitions, IoT technologies, layered architecture, Internet of Everything (IoE), visual programming and deployment of IoT, Cloud Computing, and ubiquitous cloud services feature as key aspects that promote widespread adoption and fast track learning in the field of IoT. An industrial use case for an IoT based smart fertilizer manufacturing plant was implemented using Cisco Packet Tracer 7.2-a visual tool for IoT modeling. The simulation only focused on process parameters for monitoring saturated steam temperature, converter head temperature, and neutralisation temperature respectively. The model could be extended to include key process parameters-fuel flowrates, $\mathrm{CO}$ and $\mathrm{SO}_{\mathrm{x}}$ emissions, $\mathrm{NO}_{\mathrm{x}}$ emissions, evaporator steam pressure, and other broad industrial applications. The network side can also be expanded to incorporate different levels of access control, allowing PCs in the office network to access the IoT homepage, and creating mobile applications for access on mobile devices.

\section{Acknowledgements}

Authors acknowledge Sable Chemical Industries Limited for offering their premises and staff as the research case study.

\section{Conflicts of Interest}

Authors declare no conflict of interest regarding the publication of this paper.

\section{References}

[1] Gigli, M. and Koo, S.G. (2011) Internet of Things: Services and Applications Categorization. Advances in Internet of Things, 1, 27-31. https://doi.org/10.4236/ait.2011.12004

[2] Almada-Lobo, F. (2016) The Industry 4.0 Revolution and the Future of Manufacturing Execution Systems (MES). Journal of Innovation Management, 3, 16-21. https://doi.org/10.24840/2183-0606_003.004_0003

[3] Colombo, A.W., Bangemann, T., Karnouskos, S., Delsing, J., Stluka, P., Harrison, R., Jammes, F. and Lastra, J.L. (2014) Industrial Cloud-Based Cyber-Physical Systems. The Imc-Aesop Approach, 22, 4-5.

[4] Lee, J., Bagheri, B. and Kao, H.A. (2015) A Cyber-Physical Systems Architecture for Industry 4.0-Based Manufacturing Systems. Manufacturing Letters, 3, 18-23. https://doi.org/10.1016/j.mfglet.2014.12.001

[5] Mell, P. and Grance, T. (2011) The NIST Definition of Cloud Computing. National Institute of Standards and Technology, NIST Special Publication 800-145. http://nvlpubs.nist.gov/nistpubs/Legacy/SP/nistspecialpublication800-145.pdf https://doi.org/10.6028/NIST.SP.800-145

[6] Ahmed, M. and Hossain, M.A. (2014) Cloud Computing and Security Issues in the Cloud. International Journal of Network Security \& Its Applications, 6, 25. https://doi.org/10.5121/ijnsa.2014.6103

[7] Nagy, J., Oláh, J., Erdei, E., Máté, D. and Popp, J. (2018) The Role and Impact of Industry 4.0 and the Internet of Things on the Business Strategy of the Value 
Chain-The Case of Hungary. Sustainability, 10, 3491.

https://doi.org/10.3390/su10103491

[8] Kagermann, H., Wahlster, W. and Helbig, J. (2013) Securing the Future of German Manufacturing Industry: Recommendations for Implementing the Strategic Initiative Industrie 4.0. Final Report of the Industrie 4.0 Working Group, AcatechNational Academy of Science and Engineering, $678 \mathrm{p}$.

[9] Burmeister, C., Lüttgens, D. and Piller, F.T. (2016) Business Model Innovation for Industrie 4.0: Why the "Industrial Internet" Mandates a New Perspective on Innovation. Die Unternehmung, 70, 124-152.

https://doi.org/10.5771/0042-059X-2016-2-124

[10] Tupa, J., Simota, J. and Steiner, F. (2017) Aspects of Risk Management Implementation for Industry 4.0. Procedia Manufacturing, 11, 1223-1230.

https://doi.org/10.1016/j.promfg.2017.07.248

[11] Oesterreich, T.D. and Teuteberg, F. (2016) Understanding the Implications of Digitisation and Automation in the Context of Industry 4.0: A Triangulation Approach and Elements of a Research Agenda for the Construction Industry. Computers in Industry, 83, 121-139. https://doi.org/10.1016/j.compind.2016.09.006

[12] Boeckl, K., Fagan, M., Fisher, W., Lefkovitz, N., Megas, K., Nadeau, E., Piccarreta, B., O'rourke, D.G. and Scarfone, K. (2018) Considerations for Managing Internet of Things (IoT) Cybersecurity and Privacy Risks. National Institute of Standards and Technology, NISTIR 8228 (Draft). https://doi.org/10.6028/NIST.IR.8228

[13] Hossain, M.S. and Muhammad, G. (2016) Cloud-Assisted Industrial Internet of Things (IIoT)-Enabled Framework for Health Monitoring. Computer Networks, 101, 192-202. https://doi.org/10.1016/j.comnet.2016.01.009

[14] Leitão, P., Colombo, A.W. and Karnouskos, S. (2016) Industrial Automation Based on Cyber-Physical Systems Technologies: Prototype Implementations and Challenges. Computers in Industry, 81, 11-25. https://doi.org/10.1016/j.compind.2015.08.004

[15] Patel, K.K. and Patel, S.M. (2016) Internet of Things-IOT: Definition, Characteristics, Architecture, Enabling Technologies, Application \& Future Challenges. International Journal of Engineering Science and Computing, 6, 6122-6131.

[16] Miraz, M., Ali, M., Excell, P. and Picking, R. (2018) Internet of Nano-Things, Things and Everything: Future Growth Trends. Future Internet, 10, 68. https://doi.org/10.3390/fi10080068

[17] Fisher, D.K., Fletcher, R.S. and Anapalli, S.S. (2020) Evolving Open-Source Technologies Offer Options for Remote Sensing and Monitoring in Agriculture. Advances in Internet of Things, 10, 1-10. https://doi.org/10.4236/ait.2020.101001

[18] Miraz, M.H., Ali, M., Excell, P.S. and Picking, R. (2015) A Review on Internet of Things (IoT), Internet of Everything (IoE) and Internet of Nano Things (IoNT). 2015 IEEE Internet Technologies and Applications (ITA), Wrexham, 8-11 September 2015, 219-224. https://doi.org/10.1109/ITechA.2015.7317398

[19] Tayeb, S., Latifi, S. and Kim, Y. (2017) A Survey on IoT Communication and Computation Frameworks: An Industrial Perspective. 2017 IEEE 7 th Annual Computing and Communication Workshop and Conference (CCWC), Las Vegas, 9-11 January 2017, 1-6. https://doi.org/10.1109/CCWC.2017.7868354

[20] Tanganelli, G., Mingozzi, E., Vallati, C. and Cicconetti, C. (2013) A Distributed Architecture for Discovery and Access in the Internet of Things. 2013 IEEE Conference on Computer Communications Workshops (INFOCOM WKSHPS), Turin, 14-19 April 2013, 45-46. https://doi.org/10.1109/INFCOMW.2013.6970729 
[21] Sundmaeker, H., Guillemin, P., Friess, P. and Woelfflé, S. (2010) Vision and Challenges for Realising the Internet of Things. Cluster of European Research Projects on the Internet of Things, European Commission, Luxembourg, 3, 34-36.

[22] Gubbi, J., Buyya, R., Marusic, S. and Palaniswami, M. (2013) Internet of Things (IoT): A Vision, Architectural Elements, and Future Directions. Future Generation Computer Systems, 29, 1645-1660. https://doi.org/10.1016/j.future.2013.01.010

[23] I-Scoop, 2016-2020: What Is the Internet of Things? Internet of Things Definitions. https://www.i-scoop.eu/internet-of-things

[24] Feki, M.A., Kawsar, F., Boussard, M. and Trappeniers, L. (2013) The Internet of Things: The Next Technological Revolution. Computer, 46, 24-25. https://doi.org/10.1109/MC.2013.63

[25] Bob, V. (2014) Top IT Vendors Reveal Their IoT Strategies. https://www.networkworld.com/article/2604766/top-it-vendors-reveal-their-iot-stra tegies.html

[26] Holler, J., Tsiatsis, V., Mulligan, C., Karnouskos, S., Avesand, S. and Boyle, D. (2014) Internet of Things. Academic Press, Cambridge.

[27] Donzia, S.K., Kim, H.K. and Shin, B.Y. (2018) Study on Cloud Computing and Emergence of the Internet of the Thing in Industry. 2018 IEEE 5 th NAFOSTED Conference on Information and Computer Science (NICS), Ho Chi Minh City, 23-24 November 2018, 334-337. https://doi.org/10.1109/NICS.2018.8606834

[28] Lopez Research LLC (2013) An Introduction to the Internet of Things (IoT). https://www.cisco.com/c/dam/en_us/solutions/trends/iot/introduction_to_IoT_nov ember.pdf

[29] Nikander, P., Gurtov, A. and Henderson, T.R. (2010) Host Identity Protocol (HIP): Connectivity, Mobility, Multi-Homing, Security, and Privacy over IPv4 and IPv6 Networks. IEEE Communications Surveys \& Tutorials, 12, 186-204. https://doi.org/10.1109/SURV.2010.021110.00070

[30] Li, N.P., Xiao, Y.M., Shen, L., Xu, Z.Y., Li, B.T. and Yin, C.X. (2019) Smart Agriculture with an Automated IoT-Based Greenhouse System for Local Communities. Advances in Internet of Things, 9, 15-31. https://doi.org/10.4236/ait.2019.92002

[31] IoT Agenda (2019) Internet of Things. https://internetofthingsagenda.techtarget.com/definition/Internet-of-Things-IoT

[32] Vermesan, O. and Friess, P. (2013) Internet of Things: Converging Technologies for Smart Environments and Integrated Ecosystems. River Publishers, Aalborg.

[33] Laeeq, K. and Shamsi, J.A. (2015) A Study of Security Issues, Vulnerabilities and Challenges in Internet of Things. In: Pathan, A.-S.K., Ed., Securing Cyber-Physical Systems, CRC Press, Boca Raton, 10.

[34] Jin, J., Gubbi, J., Marusic, S. and Palaniswami, M. (2014) An Information Framework for Creating a Smart City through Internet of Things. IEEE Internet of Things Journal, 1, 112-121. https://doi.org/10.1109/JIOT.2013.2296516

[35] ur Rehman, A., Khan, I.U., Murtaza, A. and Naz, U. (2018) IoT and Smart Manufacturing. In: E-Manufacturing and E-Service Strategies in Contemporary Organizations, IGI Global, Hershey, 1-38. https://doi.org/10.4018/978-1-5225-3628-4.ch001

[36] Said, O. and Masud, M. (2013) Towards Internet of Things: Survey and Future Vision. International Journal of Computer Networks, 5, 1-7.

[37] Mynzhasova, A., Radojicic, C., Heinz, C., Kölsch, J., Grimm, C., Rico, J., Dickerson, K., García-Castro, R. and Oravec, V. (2017) Drivers, Standards and Platforms for 
the IoT: Towards a Digital Vicinity. 2017 IEEE Intelligent Systems Conference (IntelliSys), London, 7-8 September 2017, 170-176. https://doi.org/10.1109/IntelliSys.2017.8324287

[38] Evans, D. (2012) The Internet of Everything: How More Relevant and Valuable Connections Will Change the World. Cisco Internet Business Solutions Group (IBSG), Cisco Systems, Inc., San Jose, 1-9.

[39] Dias, J.P. and Ferreira, H.S. (2018) State of the Software Development Life-Cycle for the Internet-of-Things.

[40] Cisco (2013).

https://www.cisco.com/c/dam/en_us/about/business-insights/docs/ioe-economy-fa q.pdf

[41] Sadeghi, A.-R., Wachsmann, C. and Waidner, M. (2015) Security and Privacy Challenges in Industrial Internet of Things. Design Automation Conference (DAC) 52nd ACM/EDAC/IEEE, San Francisco, 8-12 June 2015, 1-12. https://doi.org/10.1145/2744769.2747942

[42] Guinard, D., Trifa, V. and Wilde, E. (2010) A Resource Oriented Architecture for the Web of Things. Proceedings the 2010 Internet of Things, Tokyo, 29 November-1 December 2010, 1-8. https://doi.org/10.1109/IOT.2010.5678452

[43] Sánchez López, T., Ranasinghe, D.C., Harrison, M. and Mcfarlane, D. (2012) Adding Sense to the Internet of Things. Personal and Ubiquitous Computing, 16, 291-308. https://doi.org/10.1007/s00779-011-0399-8

[44] Gwangwava, N. (2018) Open Product Lifecycle Management (PLM) for Cloud Manufacturing and Cloud-Based Maintenance Integration Using XML. In: E-Manufacturing and E-Service Strategies in Contemporary Organizations, IGI Global, Hershey, 270-307. https://doi.org/10.4018/978-1-5225-3628-4.ch011

[45] Wei, Y., Sukumar, K., Vecchiola, C., Karunamoorthy, D. and Buyya, R. (2011) Aneka Cloud Application Platform and Its Integration with Windows Azure. CRC Press, Boca Raton.

[46] Ahmad, S., Hang, L. and Kim, D. (2018) Design and Implementation of Cloud-Centric Configuration Repository for DIY IoT Applications. Sensors, 18, 474.

https://doi.org/10.3390/s18020474

[47] Ray, P.P. (2017) A Survey on Visual Programming Languages in Internet of Things. Scientific Programming, 2017, Article ID: 1231430. https://doi.org/10.1155/2017/1231430

[48] Prehofer, C. and Chiarabini, L. (2015) From Internet of Things Mashups to Model-Based Development. 2015 IEEE 39th Annual Computer Software and Applications Conference, Vol. 3, 499-504. https://doi.org/10.1109/COMPSAC.2015.263

[49] Jost, B., Ketterl, M., Budde, R. and Leimbach, T. (2014) Graphical Programming Environments for Educational Robots: Open Roberta-Yet Another One? 2014 IEEE International Symposium on Multimedia, Taichung, 10-12 December 2014, 381-386. https://doi.org/10.1109/ISM.2014.24

[50] Azzola, F. (2018) IoT Visual Programming Tools. https://dzone.com/articles/iot-and-the-iot-visual-programming-tools

[51] Teixeira, T., Hachem, S., Issarny, V. and Georgantas, N. (2011) Service Oriented Middleware for the Internet of Things: A Perspective. In: European Conference on a Service-Based Internet, Springer, Berlin, 220-229. https://doi.org/10.1007/978-3-642-24755-2_21

[52] Giang, N.K., Blackstock, M., Lea, R. and Leung, V.C. (2015) Developing IoT Appli- 
cations in the Fog: A Distributed Dataflow Approach. 2015 IEEE 5th International Conference on the Internet of Things (IoT), Seoul, 26-28 October 2015, 155-162. https://doi.org/10.1109/IOT.2015.7356560

[53] Chatzigiannakis, I., Mylonas, G. and Nikoletseas, S. (2007) 50 Ways to Build Your Application: A Survey of Middleware and Systems for Wireless Sensor Networks. 2007 IEEE Conference on Emerging Technologies and Factory Automation (EFTA 2007), Patras, 25-28 September 2007, 466-473.

https://doi.org/10.1109/EFTA.2007.4416805

[54] Salihbegovic, A., Eterovic, T., Kaljic, E. and Ribic, S. (2015) Design of a Domain Specific Language and IDE for Internet of Things Applications. 2015 IEEE 38 th International Convention on Information and Communication Technology, Electronics and Microelectronics (MIPRO), Opatija, 25-29 May 2015, 996-1001. https://doi.org/10.1109/MIPRO.2015.7160420

[55] Kiljander, J., Takalo-Mattila, J., Etelapera, M., Soininen, J.P. and Keinanen, K. (2011) Enabling End-Users to Configure Smart Environments. 2011 IEEE/IPSJ International Symposium on Applications and the Internet, Munich, 18-21 July 2011, 303-308. https://doi.org/10.1109/SAINT.2011.58 